\title{
Problems of maximal mean resistance on the plane
}

\author{
Alexander Plakhov ${ }^{1}$ and Paulo D F Gouveia ${ }^{2}$ \\ ${ }^{1}$ Department of Mathematics, Aveiro University, Aveiro 3810, Portugal \\ 2 Techn. Manag. School, Bragança Polytechnic Institute, 5301 Bragança, Portugal
}

Received 27 March 2007, in final form 17 July 2007

Published 21 August 2007

Online at stacks.iop.org/Non/20/2271

Recommended by L Bunimovich

\begin{abstract}
A two-dimensional body moves through a rarefied medium; the collisions of the medium particles with the body are absolutely elastic. The body performs both translational and slow rotational motion. It is required to select the body, from a given class of bodies, such that the average force of resistance of the medium to its motion is maximal.

Numerical and analytical results concerning this problem are presented. In particular, the maximum resistance in the class of bodies contained in a convex body $K$ is proved to be 1.5 times the resistance of $K$. The maximum is attained on a sequence of bodies with a very complicated boundary. The numerical study was made for somewhat more restricted classes of bodies. The obtained values of resistance are slightly lower, but the boundary of obtained bodies is much simpler, as compared with the analytical solutions.
\end{abstract}

Mathematics Subject Classification: 49K30, 49Q10

(Some figures in this article are in colour only in the electronic version)

\section{Introduction}

Consider a homogeneous medium of point particles at rest in Euclidean space $\mathbb{R}^{d}$ and a body moving forward through this medium. The medium is highly rarefied, so that mutual interaction of the particles is neglected. The interaction of the particles with the body is absolutely elastic. It is required to find the shape of the body that minimizes or maximizes the resistance of the medium to its motion.

When thinking of this kind of problem, one can have in mind an artificial satellite of the Earth moving on a relatively low (say, 100-200 km) orbit; one has to minimize the resistance of the rest of the atmosphere or a solar sail, then one has to maximize the pressure of the flux of solar photons on the sail.

In order to specify the problem, one has to describe the body's motion as well as to define the class of admissible bodies. In classes of convex bodies with translational motion, the minimization problem has been extensively studied. Newton [1] obtained the solution in 
the class of (three-dimensional) convex axially symmetric bodies of fixed length and width. Since 1993, many interesting results in classes of convex non-symmetric bodies have been obtained [2-10].

Note that resistance can be written in the form $R[f]=\iint_{D}\left(1+|\nabla f|^{2}\right)^{-1} \mathrm{~d} x \mathrm{~d} y$, where the function $z=f(x, y),(x, y) \in D$ describes the front part of a convex body. Here the (orthogonal) coordinates are chosen in such a way that the body's velocity equals $(0,0,1)$. Thus, the problem amounts to minimization of the functional $R[f]$.

This approach is not valid when applied to nonconvex bodies. (A body is a bounded connected set with piecewise smooth boundary.) The reason is that the above formula is not true if particles can hit the body more than once. In fact, there is no simple analytic formula for resistance in the nonconvex case.

In general it is not easy to calculate resistance even for (nonconvex) bodies with wellbehaved boundary; however, usually one can construct a minimizing body or a minimizing sequence of bodies. In the three-dimensional case the infimum of resistance is typically equal to zero $[11,12]^{3}$. In contrast, in the two-dimensional case the infimum of resistance is positive and can usually be found explicitly [12]. In higher dimensions, $d>3$, the answer is the same as in the three-dimensional case: the infimum of resistance equals zero. Note in passing that the problem of maximal resistance admits a very simple solution: the front part of the body's surface should be orthogonal to the direction of motion or should be composed of pieces orthogonal to this direction.

It is also interesting to consider rotational motion of the body. Imagine an artificial satellite without orientation control; one can expect that in the course of motion, it will perform a (perhaps very slow) rotation. The problem of minimal mean resistance for nonconvex rotating bodies in two dimensions was considered in [13]; it was proved that the gain in resistance, as compared with the convex case, is smaller than $1.22 \%$.

Here we study the problem of maximal mean resistance for rotating bodies in two dimensions, $d=2$. This problem is far from being trivial, contrary to the case of purely translational motion. To see this, consider a unit disc on the plane, which is moving forward and at the same time slowly (and uniformly) rotating. Denote the disc by $K_{1}$. 'Cut off' a small portion of the disc contained in the $\varepsilon$-neighbourhood of $\partial K_{1}(\varepsilon \ll 1)$; the resulting set $B$ is such that $B \subset K_{1} \subset \mathcal{N}_{\varepsilon}(B)$ (here $\mathcal{N}_{\varepsilon}$ designates $\varepsilon$-neighbourhood). The question is: how large can the increase of resistance Resistance $(B) / \operatorname{Resistance}\left(K_{1}\right)$ be? Some estimates can be made immediately. Firstly, it cannot exceed 1.5. This hypothetical maximal increase is achieved if the velocity of a reflected particle is always opposite to the incidence velocity, $v^{+}=-v$; in this case the momentum transmitted by the particle to the body is maximal. Next, if the circumference $\partial K_{1}$ is partitioned into several small arcs and each arc is substituted with a pair of legs of a right isosceles triangle contained in $K_{1}$ (the resulting body is shown in figure $1(a)$ ) then the resistance increases by approximately $\sqrt{2}$ times. More precisely, if the length of each arc is $2 \varepsilon$ then the resistance increase is $\sqrt{2} \sin \varepsilon / \varepsilon \approx \sqrt{2}\left(1-\varepsilon^{2} / 6\right)$; for the proof see appendix 1.

Another example is the body obtained by making deep and narrow rectangular 'hollows' on the boundary of $K_{1}$; see figure $1(b)$. Let the depth of any hollows be $\varepsilon$, width $\varepsilon^{2}$ and the distance between neighbouring hollows $\varepsilon^{3}$. Then approximately one-half of the particles incident on a hollow escape with the velocity opposite to the initial one, and the rest of the particles escape with the velocity symmetric to the initial one with respect to the smaller side of the rectangle. Resistance of the body is, approximately, the arithmetic mean of the disc

3 This result is obtained for classes of bodies of fixed length and width [12] and for classes of bodies containing a bounded set and being contained in its $\varepsilon$-neighbourhood [11]. 


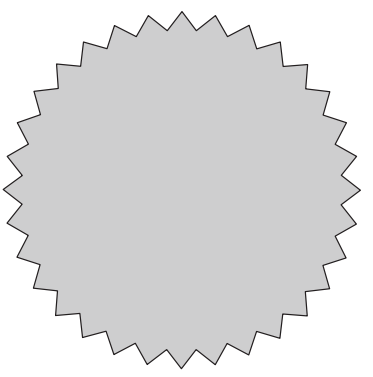

(a)

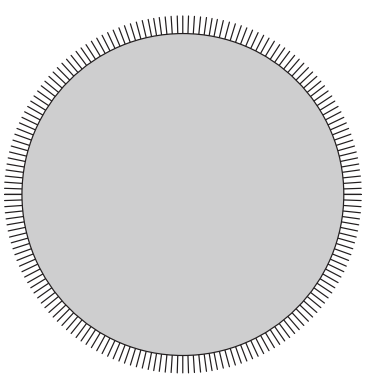

(b)

Figure 1. Two sets obtained by making small 'hollows' on the boundary of the unit circle. The hollows are $(a)$ right isosceles triangles and $(b)$ elongated rectangles.

resistance and the (hypothetical) maximal resistance (1.5 times the disc resistance); that is, the resistance increase is $1.25+o(1), \varepsilon \rightarrow 0$.

In this paper, the resistance maximization problem is studied

$(\tilde{\mathrm{A}})$ in the class of planar sets of the form $r \leqslant 1-\varepsilon f(\theta / \varepsilon), 0<\varepsilon \ll 1, f \geqslant 0$ in polar coordinates $r, \theta$ and

$(\tilde{\mathrm{B}})$ in the class of sets contained in a fixed two-dimensional convex body.

These problems still have to be rigorously stated; this is done in section 2, and the corresponding reformulated problems take the names (A) and (B). The restricted problem (A) seemed to be more amenable to numerical study and was examined first. It was not completely solved; nevertheless, we present here some numerical results. We believe that they are of interest, since they allow one to reach values of up to 1.446 (which is rather close to the upper bound of resistance) by using relatively simple geometric shapes.

When working on the restricted problem, experience was gained that eventually allowed one to solve problem (B). The answer here is 1.5 ; the corresponding maximizing sets have a much more complicated boundary than those used in (A).

The paper is organized as follows. The mathematical formulation of the problems is given in section 2. Problem (A) is studied numerically in section 3, and problem (B) is solved analytically in section 4 . The obtained results are discussed in section 5. Finally, some auxiliary formulae related to the resistance of zigzag shapes are derived in appendices 1 and 2 .

\section{Statement of the problem}

Let $B \subset \mathbb{R}^{2}$ be a bounded connected set with piecewise smooth boundary; consider the billiard in $\mathbb{R}^{2} \backslash B$. Consider a billiard particle that initially moves freely, then makes several (at least one) reflections at regular points of $\partial B$ and, finally, moves freely again. Denote by conv $B$ the convex hull of $B$.

The trajectory of the particle intersects $\partial(\operatorname{conv} B)$ twice: when entering the set $\operatorname{conv} B$ and when leaving it. Note that if the point of first intersection belongs to $I_{0}:=\partial(\operatorname{conv} B) \cap \partial B$, then the two points of intersection coincide. Introduce the natural parametrization of $\partial(\operatorname{conv} B)$ by the parameter $\xi \in[0, L]$, where $L=|\partial(\operatorname{conv} B)|$ is the length of the curve $\partial(\operatorname{conv} B)$.

Let $\xi$ and $\xi^{+}$be the first and second intersection points, and let $v$ and $v^{+}$be the particle velocity at these points, respectively. Denote by $\langle\cdot, \cdot\rangle$ the scalar product. Let $n_{\xi}$ be the outward unit normal vector to $\partial(\operatorname{conv} B)$ at the point corresponding to $\xi$. For a vector $w$ such that $\left\langle w, n_{\xi}\right\rangle \geqslant 0$, let us agree that the angle between $n_{\xi}$ and $w$ is counted from $n_{\xi}$ to $w$ clockwise 
or counterclockwise; in the first case it is positive and in the second case negative. Thus, the angle varies in the interval $[-\pi / 2, \pi / 2]$. Denote by $\varphi$ and $\varphi^{+}$the angles that the vectors $n_{\xi}$ and $n_{\xi^{+}}$form with $-v$ and $v^{+}$, respectively. (Note that one always has $n_{\xi}=n_{\xi^{+}}$.) Thereby, the one-to-one mapping $T_{B}:(\varphi, \xi) \rightarrow\left(\varphi^{+}, \xi^{+}\right)$is determined. It is defined and takes values on a full measure subset of $[-\pi / 2, \pi / 2] \times[0, L]$. Moreover, the following holds true.

(i) $T_{B}$ preserves the measure $\mu$ given by $\mathrm{d} \mu(\varphi, \xi)=\cos \varphi \mathrm{d} \varphi \mathrm{d} \xi$,

(ii) $T_{B}^{-1}=T_{B}$.

These relations follow from the measure preserving property and from time-reversibility of billiard dynamics; see [13] for more details.

Suppose now that the centre of mass of the body moves forward at a velocity $e_{2}=(0,1)$ and the body rotates around the centre of mass with a small angular velocity $\omega \ll 1$. Thus, each individual particle interacts with the body in the same way as if there were no rotation. Resistance of the medium is a periodic vector-valued function of time, $R_{B}(t)$, with the period $T=2 \pi / \omega$. In order to derive the formula for the mean value of resistance $\mathcal{R}(B)=\frac{1}{T} \int_{0}^{T} R_{B}(t) \mathrm{d} t$, consider a reference system moving forward at the velocity $e_{2}$. In this reference system, the body rotates around a fixed point, and there is a flux of particles of velocity $-e_{2}$ incident on the body. Each particle transmits to the body a momentum proportional to $v-v^{+}$, where $v=-e_{2}=(0,-1)$ is the initial velocity of the particle, $v^{+}=\left(\sin \left(\varphi^{+}-\varphi\right), \cos \left(\varphi^{+}-\varphi\right)\right)$ is its final velocity, $\varphi=\varphi_{0 i}+\omega t, t$ is the moment of the first intersection of the particle with $\partial(\operatorname{conv} B), \varphi^{+}=\varphi_{(B)}^{+}(\varphi, \xi)$; here $\xi$ means the point of the first intersection of the particle with $\partial(\operatorname{conv} B)$ and $\varphi_{(B)}^{+}(\varphi, \xi)$ is the first component of the mapping $T_{B}$. The mean resistance $\mathcal{R}(B)$ is the sum of all momenta transmitted to the body in a time interval of length $T$, divided by $T$, that is,

$\mathcal{R}(B)=-c \int_{-\pi / 2}^{\pi / 2} \int_{0}^{L}\left(\sin \left(\varphi_{(B)}^{+}(\varphi, \xi)-\varphi\right), 1+\cos \left(\varphi_{(B)}^{+}(\varphi, \xi)-\varphi\right)\right) \mathrm{d} \mu(\varphi, \xi) ;$

the ratio $c$ is proportional to the medium density. Later on we shall specify the appropriate value of $c$ simplifying the subsequent formulae.

Further, changing the variables $(\varphi, \xi) \mapsto(\tilde{\varphi}, \tilde{\xi})=\left(\varphi_{(B)}^{+}(\varphi, \xi), \xi_{(B)}^{+}(\varphi, \xi)\right)$ in integral (1) and taking into account that the Jacobian related to this change is 1 (property (i)) and that $\varphi_{(B)}^{+}(\tilde{\varphi}, \tilde{\xi})=\varphi($ property (ii)), one gets

$\mathcal{R}(B)=-c \int_{-\pi / 2}^{\pi / 2} \int_{0}^{L}\left(\sin \left(\tilde{\varphi}-\varphi_{(B)}^{+}(\tilde{\varphi}, \tilde{\xi})\right), 1+\cos \left(\tilde{\varphi}-\varphi_{(B)}^{+}(\tilde{\varphi}, \tilde{\xi})\right)\right) \mathrm{d} \mu(\tilde{\varphi}, \tilde{\xi})$.

Comparing (1) and (3) and taking into account that sine is odd, one concludes that the first component of $\mathcal{R}(B)$ is zero, that is,

$$
\mathcal{R}(B)=-c \int_{-\pi / 2}^{\pi / 2} \int_{0}^{L}\left(1+\cos \left(\varphi_{(B)}^{+}(\varphi, \xi)-\varphi\right)\right) \mathrm{d} \mu(\varphi, \xi) \cdot e_{2} .
$$

Let us now reduce formula (3) to a form more convenient for computation. The curve $\partial(\operatorname{conv} B)$ is the union of a finite or countable family of sets $I_{0}, I_{1}, I_{2}, \ldots$,

$$
\partial(\operatorname{conv} B)=\cup_{i} I_{i}
$$

Here $I_{0}=\partial(\operatorname{conv} B) \cap \partial B$ is the 'convex part' of the boundary $\partial B$ and $\partial(\operatorname{conv} B) \backslash \partial B$ is the union of open intervals $I_{1}, I_{2}, \ldots$. Respectively, $\operatorname{conv} B$ is the union of a finite or countable family of sets $\Omega_{0}, \Omega_{1}, \Omega_{2}, \ldots$,

$$
\operatorname{conv} B=\cup_{i} \Omega_{i},
$$




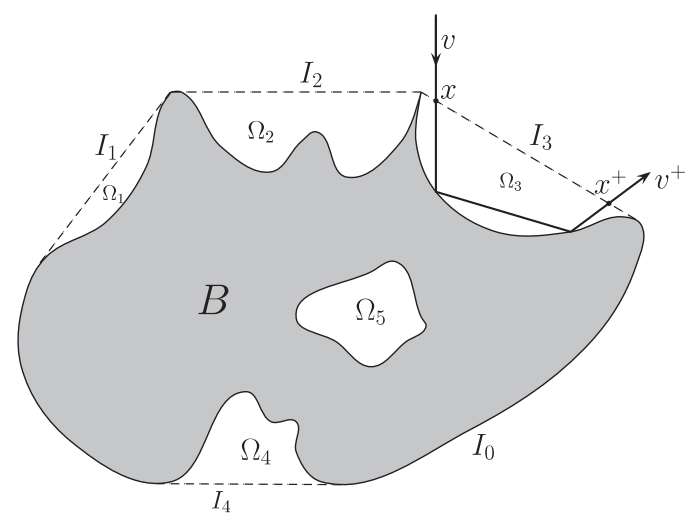

Figure 2. A billiard trajectory in $\mathbb{R}^{2} \backslash B$.

where $\Omega_{0}=B$ and the sets $\Omega_{1}, \Omega_{2}, \ldots$ are connected components of conv $B \backslash B$ ('cavities' on $B$ ). The enumeration is chosen in such a way that $I_{i} \subset \partial \Omega_{i}$ (see figure 2). Note that for some sets $\Omega_{i}$ there may occur $\partial \Omega_{i} \subset \partial B$. These sets ('interior cavities' of $B$ ) have no influence on resistance and will be ignored in what follows.

Each interval $I_{i}(i \neq 0)$ corresponds to an interval $\mathcal{L}_{i}$ (modulo L) on the parameter set $[0, L]$, and $I_{0}$ corresponds to $[0, L] \backslash \cup_{i \neq 0} \mathcal{L}_{i}=: \mathcal{L}_{0}$. Let $l_{i}$ be the length of $L_{i}$. The sets $[-\pi / 2, \pi / 2] \times \mathcal{L}_{i}$ are invariant with respect to $T_{B}$; denote by $T_{i}$ the restrictions of $T_{B}$ on these sets and by $\varphi_{i}(\varphi, \xi), \xi_{i}(\varphi, \xi)$, the components of $T_{i}$. One easily sees that $T_{0}$ is given by $T_{0}(\varphi, \xi)=(-\varphi, \xi)$. The mean resistance $\mathcal{R}(B)(3)$ is the sum of partial resistances $\mathcal{R}_{i}$ related to the $i$ th cavity: $\mathcal{R}(B)=\sum_{i} \mathcal{R}_{i}$, where

$$
\mathcal{R}_{i}=-c \int_{-\pi / 2}^{\pi / 2} \int_{\mathcal{L}_{i}}\left(1+\cos \left(\varphi_{i}^{+}(\varphi, \xi)-\varphi\right)\right) \mathrm{d} \mu(\varphi, \xi) \cdot e_{2} .
$$

In particular,

$$
\mathcal{R}_{0}=-c \int_{-\pi / 2}^{\pi / 2} \int_{\mathcal{L}_{0}}(1+\cos 2 \varphi) \cos \varphi \mathrm{d} \varphi \mathrm{d} \xi \cdot e_{2}=-\frac{8}{3} c l_{0} e_{2} .
$$

Put $c=3 / 8$, then one gets a slightly simplified relation: $\mathcal{R}_{0}=-l_{0} e_{2}$.

We say that a bounded set $\Omega \subset \mathbb{R}^{2}$ with piecewise smooth boundary is a standard cavity if it contains the interval $\mathcal{I}:=[0,1] \times\{0\}$ and is contained in the upper half-plane $\left\{\left(x_{1}, x_{2}\right): x_{2} \geqslant 0\right\}$, that is,

$$
\mathcal{I} \subset \Omega \subset\left\{\left(x_{1}, x_{2}\right): x_{2} \geqslant 0\right\} .
$$

Consider a billiard in $\Omega$; suppose that a billiard particle starts from a point of $\mathcal{I}$ and after several reflections from $\partial \Omega \backslash \mathcal{I}$ returns to $\mathcal{I}$. Let $(\xi, 0)$ and $(\sin \varphi, \cos \varphi)$ be the initial location and initial velocity of the particle and denote by $\left(\xi_{\Omega}(\varphi, \xi), 0\right)$ and $-\left(\sin \varphi_{\Omega}(\varphi, \xi), \cos \varphi_{\Omega}(\varphi, \xi)\right)$ the final point and final velocity (see figure 3$)$. The so-defined map $(\varphi, \xi) \mapsto\left(\varphi_{\Omega}(\varphi, \xi), \xi_{\Omega}(\varphi, \xi)\right)$ preserves the measure $\mu$ and is defined and takes values on a full measure subset of $[-\pi / 2, \pi / 2] \times[0,1]$.

Designate

$$
\mathcal{F}(\Omega)=\frac{3}{8} \int_{-\pi / 2}^{\pi / 2} \int_{0}^{1}\left(1+\cos \left(\varphi_{\Omega}(\varphi, \xi)-\varphi\right)\right) \cos \varphi \mathrm{d} \xi \mathrm{d} \varphi ;
$$

The integrand in (5) does not exceed 2 ; therefore $\mathcal{F}(\Omega) \leqslant(3 / 8) \int_{-\pi / 2}^{\pi / 2} \int_{0}^{1} 2 \cos \varphi \mathrm{d} \xi \mathrm{d} \varphi=1.5$. On the other hand, denoting $\Omega_{\varepsilon}:=\pi_{\varepsilon} \Omega$, where $\pi_{\varepsilon}:\left(x_{1}, x_{2}\right) \mapsto\left(x_{1}, \varepsilon x_{2}\right)$, one 


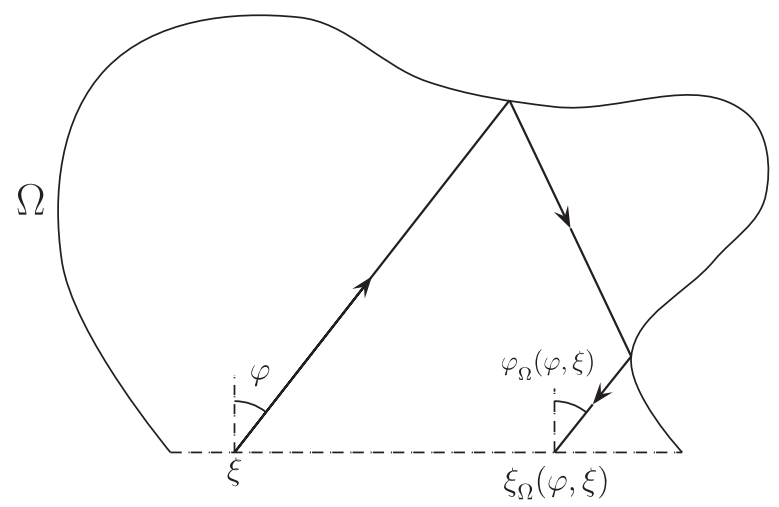

Figure 3. A standard cavity.

has that $\varphi_{\Omega_{\varepsilon}}(\varphi, \xi) \rightarrow-\varphi$ as $\varepsilon \rightarrow 0^{+}$; hence $\lim _{\varepsilon \rightarrow 0^{+}} \mathcal{F}\left(\Omega_{\varepsilon}\right)=(3 / 8) \int_{-\pi / 2}^{\pi / 2} \int_{0}^{1}(1+$ $\cos 2 \varphi) \cos \varphi \mathrm{d} \xi \mathrm{d} \varphi=1$. It follows that $1 \leqslant \sup _{\Omega} \mathcal{F}(\Omega) \leqslant 1.5$.

Each pair $\left(\Omega_{i}, I_{i}\right), i=1,2, \ldots$ can be reduced, by a similarity transformation and a translation, to the form $\left(\tilde{\Omega}_{i}, \mathcal{I}\right)$, where $\tilde{\Omega}_{i}$ is a standard cavity. Denote $\lambda_{i}=l_{i} /|\partial(\operatorname{conv} B)|$; one has $\sum_{i} \lambda_{i}=1$ and $\mathcal{R}_{i}=-\lambda_{i} \mathcal{F}\left(\tilde{\Omega}_{i}\right)$, therefore

$$
\mathcal{R}(B)=-|\partial(\operatorname{conv} B)| \cdot\left(\lambda_{0}+\sum_{i \neq 0} \lambda_{i} \mathcal{F}\left(\tilde{\Omega}_{i}\right)\right) \cdot e_{2} .
$$

Let the set $B_{\varepsilon}$ be given by $r \leqslant 1-\varepsilon f(\theta / \varepsilon)$ in polar coordinates $r, \theta$, where $f$ is a 1-periodic continuous piecewise differentiable nonnegative function and $\varepsilon$ divides $2 \pi$. Suppose that $f(0)=f(1)=0$. As $\varepsilon \rightarrow 0, \mathcal{R}\left(B_{\varepsilon}\right)$ tends to $-2 \pi \cdot \mathcal{F}\left(\Omega_{f}\right)$, where

$$
\Omega_{f}=\left\{\left(x_{1}, x_{2}\right): 0 \leqslant x_{1} \leqslant 1,0 \leqslant x_{2} \leqslant f\left(x_{1}\right)\right\} .
$$

Thus, problem ( $\tilde{\mathrm{A}})$ (which has not yet been rigorously formulated) can be stated as follows.

(A) Find $\sup _{f} \mathcal{F}\left(\Omega_{f}\right)$ over all continuous piecewise differentiable nonnegative functions $f:[0,1] \rightarrow \mathbb{R}_{+}$such that $f(0)=f(1)=0$.

Problem $(\tilde{\mathrm{B}})$ reads as: find $\sup _{B \subset K}|\mathcal{R}(B)|$, where $K \subset \mathbb{R}^{2}$ is a convex bounded set with nonempty interior. In view of (6), it amounts to the problem

(B) Find $\sup _{\Omega} \mathcal{F}(\Omega)$ over all standard cavities $\Omega$.

Indeed, let $\Omega_{n}$ be a sequence of sets solving problem (B); then a sequence of bodies $B_{n} \subset K$ approximating $K$ solves problem $(\tilde{\mathrm{B}})$, if all the cavities of $B_{n}$ are similar to $\Omega_{n}$ and the length of the convex part of $B_{n}$ tends to zero. Thus, one has $\sup _{B \subset K}|\mathcal{R}(B)|=|\partial K| \cdot \sup _{\Omega} \mathcal{F}(\Omega)$.

\section{Problem (A): numerical results}

Introduce the shorthand notation $\mathcal{F}[f]:=\mathcal{F}\left(\Omega_{f}\right)$. Note that the functional $\mathcal{F}$ is continuous in the $C^{1}$ topology: if $f_{n} \stackrel{C^{1}}{\longrightarrow} f$ then $\mathcal{F}\left[f_{n}\right] \rightarrow \mathcal{F}[f]$; thus there exists a sequence of piecewise linear functions maximizing $\mathcal{F}$. Therefore, it seems natural to look for the maximum in classes of continuous functions $f$ with piecewise constant derivative $f^{\prime}$. We also examined classes of continuous functions with piecewise constant second derivative $f^{\prime \prime}$. In the first case the graph of $f$ is a broken line and in the second a curve composed of arcs of parabolas. 


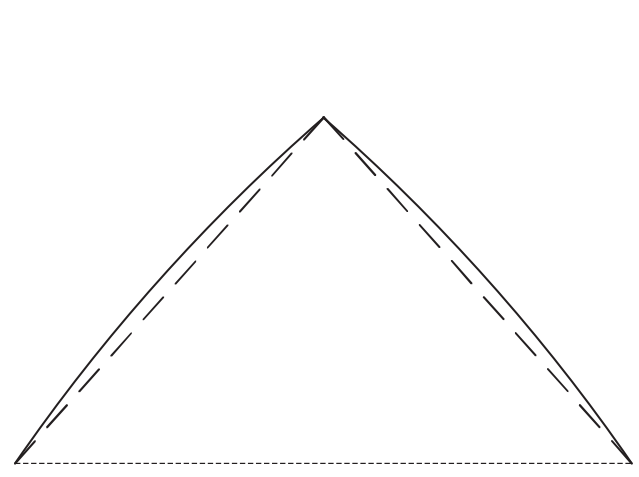

(a)

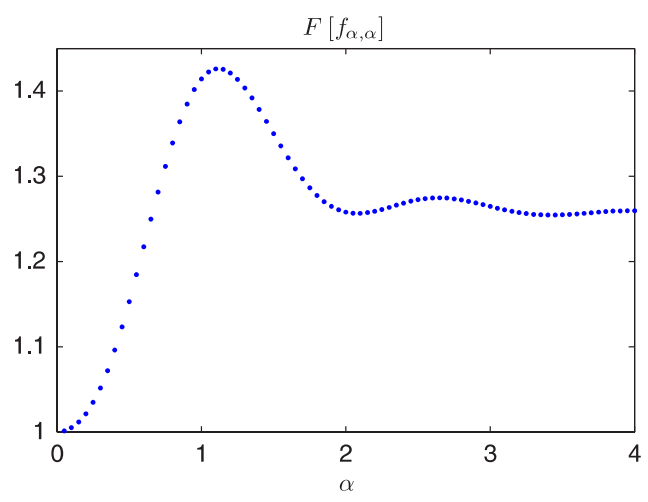

(b)

Figure 4. (a) The solutions of problem (A) in the class of two-segment piecewise linear functions (- - -) and two-segment piecewise quadratic functions (- $) ;(b)$ graph of the function $F\left[f_{\alpha, \alpha}\right]$.

In each numerical experiment $N_{1} N_{2}$ trials with a billiard particle in $\Omega_{f}$ were made. Usually $N_{1}$ and $N_{2}$ were taken equal and varied from several hundreds to several thousands (up to 5000). Initially, the particle is located at $\left(\xi_{i}, 0\right) \in \mathcal{I}$, where $\xi_{i}=(i-1 / 2) / N_{1}$, and has the velocity $\left(\sin \varphi_{j}, \cos \varphi_{j}\right)$, where $\varphi_{j}=\pi\left(j-\frac{N_{2}+1}{2}\right) / N_{2}, i=1, \ldots, N_{1}, j=1, \ldots, N_{2}$. Then the least time instant, when the particle gets into $\mathcal{I}$ again, is fixed, and the particle velocity $v_{i j}=-\left(\sin \varphi_{i j}^{+}, \cos \varphi_{i j}^{+}\right)$just before this instant is registered. The sum

$$
\mathcal{F}=\frac{3}{8} \frac{\pi}{N_{1} N_{2}} \sum_{i=1}^{N_{1}} \sum_{j=1}^{N_{2}} \cos \varphi_{j}\left(1+\cos \left(\varphi_{j}-\varphi_{i j}^{+}\right)\right)
$$

is considered to be an approximation for the integral $\mathcal{F}[f]$.

The algorithm simulating the billiard dynamics, as well as numerical integration, was implemented in programming language $\mathrm{C}$. The precision accuracy achieved $10^{-6}$; it was controlled, firstly, by the differences between the successive approximations of $\mathcal{F}$ as $N_{1}$ and $N_{2}$ increased and secondly by comparison with the analytic results. To maximize the resistance, optimization algorithms from the Genetic Algorithm and Direct Search Toolbox of the computational system MATLAB, version 7.2, were used; these methods do not require any information on the derivatives of the objective function.

The obtained results are as follows.

1. In the class of two-segment broken lines

$$
f_{\alpha, \beta}(\xi)= \begin{cases}\alpha \xi, & \text { if } 0 \leqslant \xi \leqslant \xi_{0} \\ \beta(1-\xi), & \text { if } \xi_{0} \leqslant \xi \leqslant 1\end{cases}
$$

where $\alpha>0, \beta>0,0<\xi_{0}<1, \alpha \xi_{0}=\beta\left(1-\xi_{0}\right)$, the maximum of $\mathcal{F}$ equals 1.42621 and is achieved at $\alpha=\beta=\alpha_{0} \approx 1.12$. Then $\xi_{0}=0.5$; the corresponding set $\Omega_{f_{\alpha_{0}, \alpha_{0}}}$ is an isosceles triangle with the angle $83.6^{\circ}$ at the top vertex. It is shown in figure $4(a)$, with the lateral sides drawn dashed.

The function $\mathcal{F}\left[f_{\alpha, \alpha}\right]$ oscillates and goes to 1.25 as $\alpha \rightarrow+\infty$ (see figure $4(b)$ ). 2 . In the class of two-segment piecewise quadratic functions

$$
f_{\alpha_{1} \alpha_{2} \beta_{1} \beta_{2}}(\xi)= \begin{cases}\alpha_{1} \xi^{2}+\beta_{1} \xi, & \text { if } 0 \leqslant \xi \leqslant \xi_{0} \\ \alpha_{2}(1-\xi)^{2}+\beta_{2}(1-\xi), & \text { if } \xi_{0} \leqslant \xi \leqslant 1\end{cases}
$$




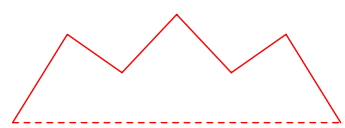

(a) $m=6(1.444983)$

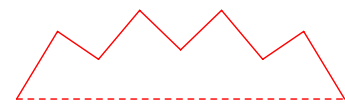

(b) $m=8(1.445562)$

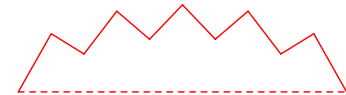

(c) $m=10(1.446227)$

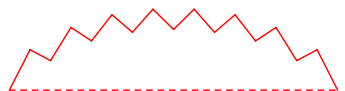

(d) $m=16(1.445535)$

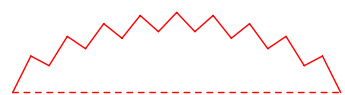

(e) $m=18(1.445931)$

Figure 5. The functions providing the largest value for the functional $\mathcal{F}$ found in the class of $m$-segment broken lines: numerical solutions for $m=6,8,10,16$ and 18 are shown.

where $0<\xi_{0}<1, \alpha_{1} \xi_{0}^{2}+\beta_{1} \xi_{0}=\alpha_{2}\left(1-\xi_{0}\right)^{2}+\beta_{2}\left(1-\xi_{0}\right)$, the maximum of $\mathcal{F}$ is achieved at $\xi_{0}=0.5, \alpha_{1}=\alpha_{2}=\alpha \approx-0.486, \beta_{1}=\beta_{2}=\beta \approx 1.361$ and is equal to 1.43816 . The corresponding set $\Omega_{f_{\alpha \alpha \beta \beta}}$ is a curvilinear isosceles triangle; it is shown in figure 4(a) with lateral sides drawn with solid lines. Its height is equal to the height of the optimal triangle from item 1; so to say, this triangle is obtained from the previous one by a slight 'bending outwards' of its lateral sides.

3. In the class of broken lines with many segments the simulations become more cumbersome. Let $x^{0}=\left(x_{1}^{0}, x_{2}^{0}\right)=(0,0), x^{1}=\left(x_{1}^{1}, x_{2}^{1}\right), \ldots, x^{m}=\left(x_{1}^{m}, x_{2}^{m}\right)=(1,0)$ be the vertices of the broken line, with $m$ being the number of segments. Experiments with relatively small $m(m \leqslant 5)$ showed that making the broken line symmetric with respect to the vertical line $x_{1}=1 / 2$ and taking the values $x_{1}^{i}, i=1, \ldots, m-1$ equally spaced in $[0,1]$ favour some moderate increase of resistance. Therefore, the posterior study was restricted to symmetric broken lines with $x_{1}^{i}=i / m$, and thus the number of effective parameters was reduced by almost four times: from $2(m-1)$ to $\left\lfloor\frac{m}{2}\right\rfloor$. This decision allowed one to take a relatively large number of segments, $1 \leqslant m \leqslant 18$.

Many 'zigzag' shapes providing nearly maximal values $\mathcal{F} \approx 1.446 \ldots$ were found; some typical shapes are shown in figure 5. The greatest found value of $\mathcal{F}$ corresponds to the shape shown in figure $5(c)(m=10)$ and equals 1.446227.

In order to verify the simulation results, as well as to find out the common analytic form of the obtained curves, we examined the following broken line. Fix $\Psi \in[0, \pi / 2]$ and consider the arc of angular size $2 \Psi$ contained in the upper half-plane $x_{2} \geqslant 0$, with the endpoints $(0,0)$ and $(1,0)$. Let $m$ be even. Mark the points $x^{0}=\left(x_{1}^{0}, x_{2}^{0}\right)=(0,0)$, $x^{2}=\left(x_{1}^{2}, x_{2}^{2}\right), \ldots, x^{2 i}=\left(x_{1}^{2 i}, x_{2}^{2 i}\right), \ldots, x^{m}=\left(x_{1}^{m}, x_{2}^{m}\right)=(1,0)$ on the arc, with $0=x_{1}^{0}<x_{1}^{2}<\cdots<x_{1}^{m}=1$. Let $\delta$ be the maximum of values $x_{1}^{2 i}-x_{1}^{2 i-2}$. We say that a right triangle $A B C$ is canonical, if vertex $B$ is situated above the hypotenuse $A C$ and the median drawn from $B$ to $A C$ is vertical. For $i=1, \ldots, m / 2$, draw the canonical triangle $\triangle x^{2 i-2} x^{2 i-1} x^{2 i}$ with the hypotenuse $\left[x^{2 i-2}, x^{2 i}\right]$. Thus, one has $x_{1}^{2 i-1}=\frac{1}{2}\left(x_{1}^{2 i-2}+x_{1}^{2 i}\right)$, $x_{2}^{2 i-1}=\frac{1}{2}\left(x_{2}^{2 i-2}+x_{2}^{2 i}\right)+\frac{1}{2} \sqrt{\left(x_{1}^{2 i}-x_{1}^{2 i-2}\right)^{2}+\left(x_{2}^{2 i}-x_{2}^{2 i-2}\right)^{2}}$. The broken line $x^{0} x^{1} \ldots x^{m-1} x^{m}$ composed of legs of all triangles obtained this way will also be called canonical (the graphs shown in figure 5 are good approximations for canonical lines with $m=6,8,10,16,18$ ). If $\Psi$ is fixed and $\delta$ goes to zero, the corresponding value of $\mathcal{F}$ tends to

$$
\mathcal{F}(\Psi)=1+\frac{1}{6} \sin ^{2} \Psi+\frac{2 \sqrt{2} \sin \frac{\Psi}{2}-2 \sin ^{4} \frac{\Psi}{2}-\Psi}{\sin \Psi}
$$

(here $\Psi$ is expressed in radians). The proof of this convergence is given in appendix 2 . The maximal value of $\mathcal{F}(\Psi)$ is achieved at $\Psi_{0} \approx 0.6835 \approx 39.16^{\circ}$ and is equal to $\mathcal{F}\left(\Psi_{0}\right)=1.445209$. 


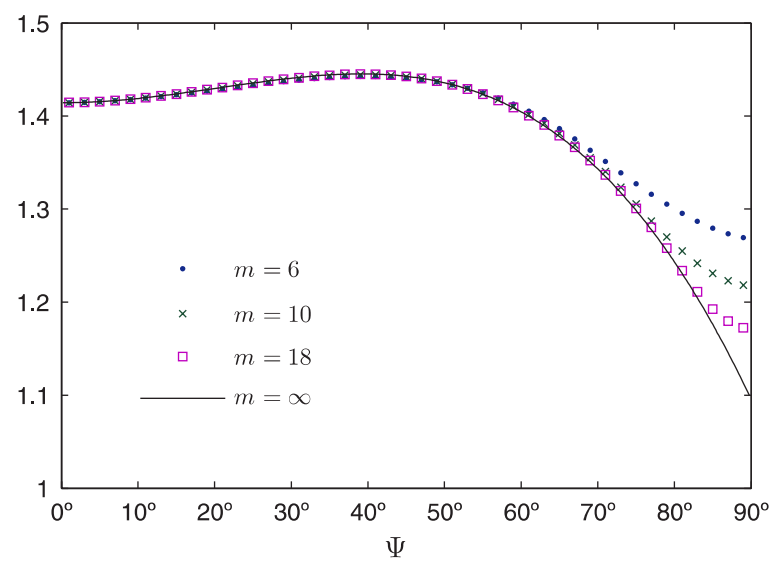

Figure 6. The functions $\mathcal{F}_{m}(\Psi)$, with $m=6,10$ and 18, and the limit function $\mathcal{F}(\Psi)(m=\infty)$ are shown.

Let $\mathcal{F}_{m}(\Psi)$ be the values of $\mathcal{F}$ related to the canonical lines with $x_{1}^{i}=i / m, i=0, \ldots, m$. These values were numerically calculated for various values of $\Psi$ and for $m=6,10,18$. The resulting functions $\mathcal{F}_{m}(\Psi)$ and the function $\mathcal{F}(\Psi)$ (solid line) are shown in figure 6 . Note in passing that in the limit $\Psi \rightarrow 0$ the triangles of the corresponding canonical line approach a right isosceles triangle; therefore, the corresponding value of $\mathcal{F}$ tends to $\sqrt{2}$; that is, $\mathcal{F}(0)=\sqrt{2}$.

4. In the class of piecewise quadratic functions, with $\leqslant 18$ segments, the greatest found value of $\mathcal{F}$ is 1.44772 ; this is the maximal value found numerically up to the moment. The corresponding curve is a 14-segment 'zigzag' line with slightly concave segments; its visual perception is the same as of the curves shown in figure 5 .

5. We examined numerically shapes formed by infinitely small canonical triangles. In these experiments we had to substitute the usual billiard dynamics with the pseudo-billiard one described in appendix 2. Also, we tried shapes formed by various kinds of non-canonical triangles. However, we could not increase the resistance this way.

Thus, one has the following estimates for problem (A):

$$
1.445 \leqslant \sup _{f} \mathcal{F}\left(\Omega_{f}\right) \leqslant 1.5 \text {. }
$$

The value 1.445 (which is slightly less than the better numerical value $1.447 \ldots$. ) is justified and explained analytically.

\section{Solution of problem (B)}

Consider the standard cavity $\Omega_{\varepsilon}=\Omega_{\varepsilon}^{\prime} \cup \Omega_{\varepsilon}^{\prime \prime}$, where $\Omega_{\varepsilon}^{\prime}=[0,1] \times[0, \varepsilon]$ is a rectangle and $\Omega_{\varepsilon}^{\prime \prime}$ is the upper semi-ellipse with the foci $F_{1}=(0, \varepsilon)$ and $F_{2}=(1, \varepsilon)$ and with the major semi-axis of length $1 / \varepsilon$. This figure is a mushroom, with the stem $\Omega_{\varepsilon}^{\prime}$ and the cap $\Omega_{\varepsilon}^{\prime \prime}$ (see figure 7). Note that mushroom was first proposed by Bunimovich as an example of a billiard with divided phase space [14].

Consider the billiard particles starting from $\mathcal{I}=[0,1] \times\{0\}$. These particles, except for a small part of them, make a unique reflection from the elliptical arc and then return to $\mathcal{I}$, the angle between the initial and final velocity being less than $2 \arctan (\varepsilon / 2)$. The rest of the particles, i.e. those that make reflections from the vertical sides of the stem, have the total 


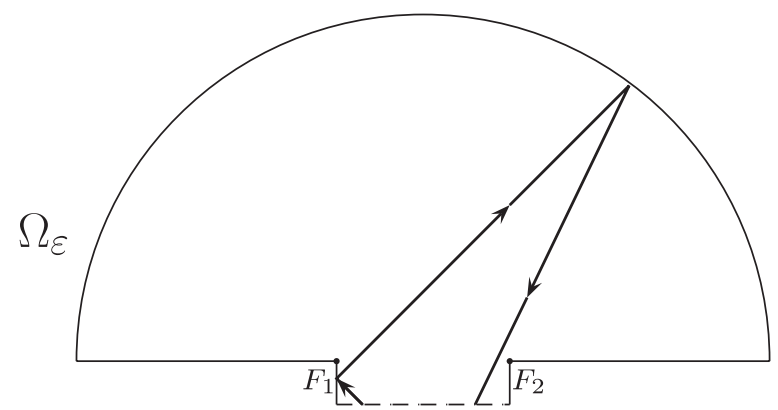

Figure 7. A mushroom.

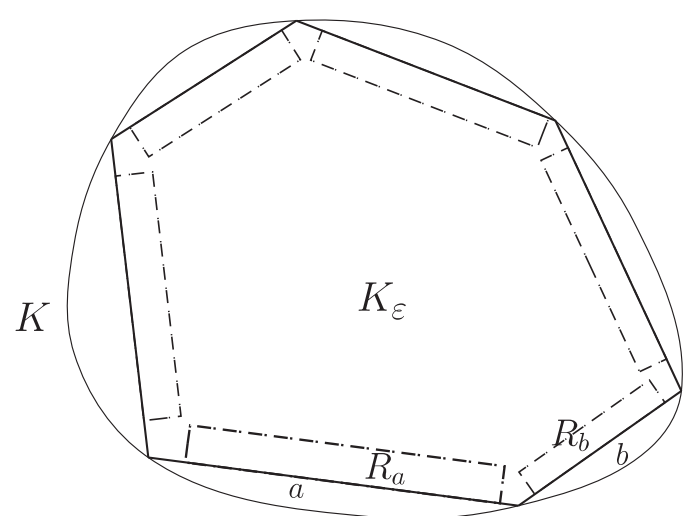

Figure 8. Construction of a body of maximal resistance. Step 1: constructing the polygon $K_{\varepsilon}$ and the rectangles $R_{a}, R_{b}, \ldots$

measure $O(\varepsilon)$. Indeed, one easily calculates that the measure of the particles having the first reflection from the stem is

$$
2 \cdot \int_{0}^{1} \mathrm{~d} \xi \int_{\arctan (\xi / \varepsilon)}^{\pi / 2} \cos \varphi \mathrm{d} \varphi=2\left(1+\varepsilon-\sqrt{1+\varepsilon^{2}}\right) .
$$

The measure of the particles having at least one reflection from a stem size is at most double this value.

Taking into account that the total measure of all particles incident on $\mathcal{I}$ is $\int_{-\pi / 2}^{\pi / 2} \int_{0}^{1} \cos \varphi \mathrm{d} \xi \mathrm{d} \varphi=2$ and using (5), one gets

$\mathcal{F}\left(\Omega_{\varepsilon}\right) \geqslant \frac{3}{8}\left(2-4\left(1+\varepsilon-\sqrt{1+\varepsilon^{2}}\right)\right)\left(1+\cos \left(2 \arctan \frac{\varepsilon}{2}\right)\right)=1.5+O(\varepsilon)$.

Thus, problem $(\mathrm{B})$ is solved: $\sup _{\Omega} \mathcal{F}(\Omega)=1.5$.

Now let $K$ be a convex bounded body with a nonempty interior. Approximate it by a convex polygon $K_{\varepsilon} \subset K, \varepsilon>0$ such that $|\partial K|-\left|\partial K_{\varepsilon}\right|<\varepsilon$. To each side $a, b, \ldots$ of $K_{\varepsilon}$ assign a rectangle $R_{a}, R_{b}, \ldots$ such that one side of the rectangle (denote it by $a^{\prime}, b^{\prime}, \ldots$, respectively) belongs to $a, b, \ldots$, etc; all the rectangles belong to $K_{\varepsilon}$ and do not mutually intersect, and the common length of the part of perimeter of $K_{\varepsilon}$ not occupied by $a^{\prime}, b^{\prime}, \ldots$, is less than $\varepsilon$ (see figure 8). On each rectangle plant out 'seedlings of mushrooms', as shown in figure $9(a)$ for $R_{a}$. The sides of $R_{a}$ that do not belong to $a$ are shown dashed. In figure $9(a), R_{a} \backslash$ (union of mushrooms) is shown, all the mushrooms being of equal size and similar to $\Omega_{\varepsilon}$. The total 


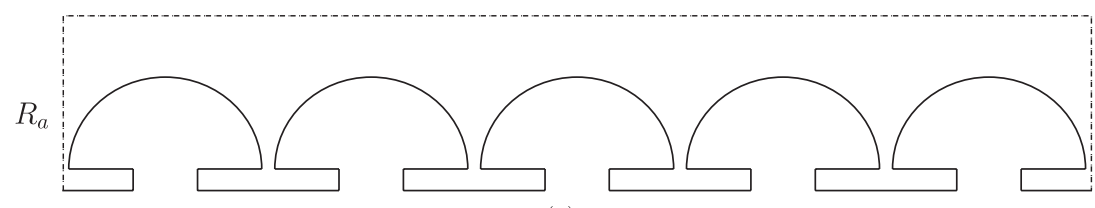

(a)

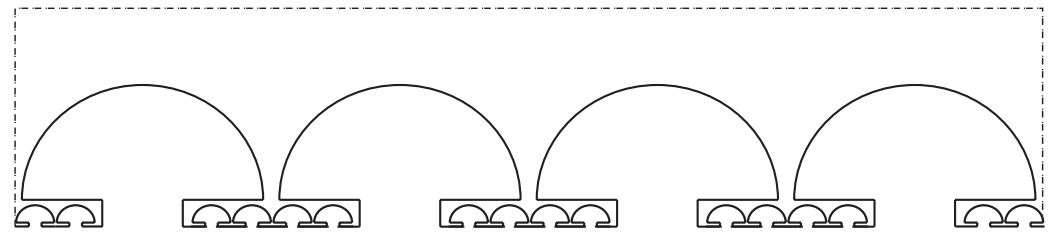

(b)

Figure 9. Construction of a body of maximal resistance. Step 2: planting out seedlings of mushrooms of the first order $(a)$ and of the second order $(b)$.

length of the lower horizontal part (1.h.p.) of the boundary of the obtained figure is $1-\varepsilon$ times the length of the corresponding size of $R_{a}$, that is, $(1-\varepsilon)\left|a^{\prime}\right|$.

Now, plant out 'seedlings' of 'mushrooms of the second order' (see figure $9(b)$ ). Here the length of l.h.p. of the boundary is $(1-\varepsilon)^{2}\left|a^{\prime}\right|$. Continuing this process, one finally obtains the figure $\tilde{R}_{a}$ such that the length of the 1.h.p. of its boundary is less than $\varepsilon\left|a^{\prime}\right|$. Similarly, one obtains the figures $\tilde{R}_{b}, \ldots$

Put $B_{\varepsilon}=\left(K_{\varepsilon} \backslash\left(R_{a} \cup R_{b} \cup \ldots\right)\right) \cup\left(\tilde{R}_{a} \cup \tilde{R}_{b} \cup \ldots\right)$. All the cavities of $B_{\varepsilon}$ are similar to $\Omega_{\varepsilon}$, the length of the convex part of $\partial B_{\varepsilon}$ tends to zero and $\left|\partial\left(\operatorname{conv} B_{\varepsilon}\right)\right| \rightarrow|\partial K|$ as $\varepsilon \rightarrow 0$. (Recall that the convex part of $\partial B_{\varepsilon}$ is $\partial B_{\varepsilon} \cap \partial\left(\operatorname{conv} B_{\varepsilon}\right)$.) Therefore $\lim _{\varepsilon \rightarrow 0}\left|\mathcal{R}\left(B_{\varepsilon}\right)\right|=1.5|\mathcal{R}(K)|$; this gives the supremum of resistance for problem $(\tilde{\mathrm{B}})$.

If $K$ is not a polygon, the solution of this problem does not exist. Instead, the supremum is attained at a family of bodies approximating $K$, with the size of cavities going to zero. The same is true for problem $(\tilde{\mathrm{A}})$.

The existence of a solution for problems (A) or (B) (and for problem ( $\tilde{\mathrm{B}})$ where $K$ is a polygon) means the existence of a cavity reversing the direction of motion of all incident particles. The question remains open, but we think the existence is unlikely. An issue more important for applications is searching for simple shapes that perform an approximate reversion of the direction of incident particles.

\section{Conclusions}

There are many technical devices utilizing wind pressure force: for example, ship sail and windmill arm. We are interested here in maximizing the pressure force of the wind consisting of noninteracting particles. An example of such a wind is provided by the flow of solar photons incident on a solar sail. We first studied the problem numerically and in a restricted class of bodies and then, based on the gained experience and intuition, found the solution analytically.

In figure 10, 'pre-optimal' bodies are shown, that is, elements of maximizing sequences of bodies for problems $(\tilde{\mathrm{A}})$ and $(\tilde{\mathrm{B}})$. (In case $(\tilde{\mathrm{B}}), K$ is the unit circle.) One can see that the boundary of the second figure is much more complicated than that of the first one. The 'complexity' of the boundary can be measured by the maximal value of the rotation 


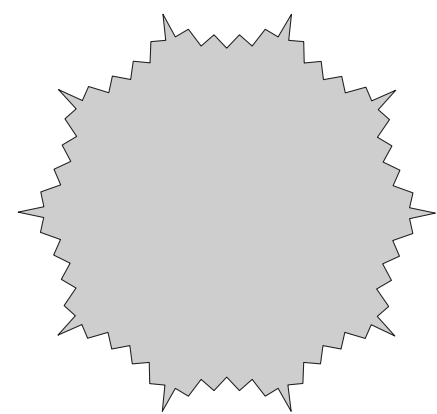

(a)

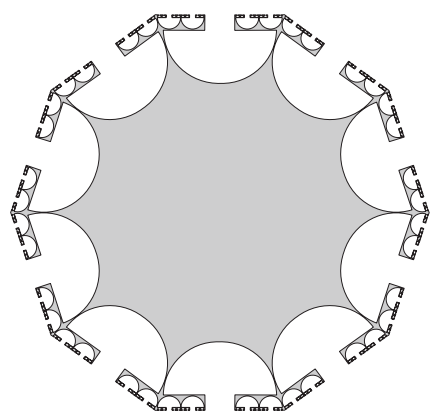

(b)

Figure 10. 'Pre-optimal bodies' for problems $(\tilde{\mathrm{A}})$ and $(\tilde{\mathrm{B}})$.

angle of the normal vector on a small part of the boundary. This 'maximal instantaneous rotation' is, approximately, $90^{\circ}+\Psi_{0} \approx 129^{\circ}$ for the first figure and $360^{\circ}$ for the second one.

To be precise, fix a convex bounded body $K$ and consider a sequence $B_{n}, n=1,2, \ldots$ approximating $K$. Define the value $\operatorname{rot}\left(\left\{B_{n}\right\}\right)$ measuring the boundary complexity of the sequence in the following way. For each point $x \in \partial B_{n}$, let $v_{x}^{n}$ be the outer unit normal at $x$ and let $\theta_{n}(x):=\arg \left(v_{x}^{n}\right)$. For any $x \in \partial K, \operatorname{define} \operatorname{rot}_{n}(x, \varepsilon):=\sup _{x_{1}, x_{2} \in \partial B_{n} \cap \mathcal{N}_{\varepsilon}(x)}\left(\theta_{n}\left(x_{2}\right)-\theta_{n}\left(x_{1}\right)\right) ;^{4}$ let us call it the $(x, \varepsilon)$-rotation of the boundary $\partial B_{n}$. It is monotone non-increasing as $\varepsilon \rightarrow 0$. Then put $\operatorname{rot}_{n}(\varepsilon)=\sup _{x \in \partial K} \operatorname{rot}_{n}(x, \varepsilon)$ and define $\operatorname{rot}(\varepsilon)=\lim \sup _{n \rightarrow \infty} \operatorname{rot}_{n}(\varepsilon): \varepsilon$-rotation for the approximating sequence $B_{n}$. Finally, define $\operatorname{rot}\left(\left\{B_{n}\right\}\right)=\lim _{\varepsilon \rightarrow 0} \operatorname{rot}(\varepsilon)$ and call this value rotation of boundary for the given sequence of bodies. This value is $\pi / 2+\Psi_{0}$ for the first sequence of figures and $2 \pi$ for the second one. The difference is almost threefold.

Further, the boundary length for the second sequence of figures tends to infinity. On the other hand, the boundary length tends to $\frac{\sqrt{2}}{\cos \left(\Psi_{0} / 2\right)} 2 \pi=1.501 \cdot 2 \pi$ for the first sequence; that is, the limit value is approximately 1.5 times the perimeter of unit circle.

Throughout this paper it was assumed that the 'inner temperature' of the wind is zero. However, one can show that in the case of positive temperature, that is, the chaotic relative motion of wind particles, the functional to be minimized is proportional to the functional $\mathcal{F}$ (5). Therefore, all the results of this paper remain valid in the case of positive temperature; the detailed explication is postponed to a future paper. We will also study the three-dimensional case in a future paper.

\section{Acknowledgment}

This work was supported by the Centre for Research on Optimization and Control (CEOC) from the 'Fundação para a Ciência e a Tecnologia' (FCT), cofinanced by the European Community Fund FEDER/POCTI.

\section{Appendix 1}

Denote by $B^{(\varepsilon)}$ the set depicted in figure $1(a)$ in the introduction. Here $\varepsilon$ divides $\pi$ and $\operatorname{conv} B^{(\varepsilon)}$ is a $(\pi / \varepsilon)$-sided regular polygon inscribed in the unit circle $K_{1}$. Its perimeter is $\left|\partial\left(\operatorname{conv} B^{(\varepsilon)}\right)\right|=2 \pi \sin \varepsilon / \varepsilon$. The convex part of $\partial B^{(\varepsilon)}$ is a finite collection of points and all the cavities of $B^{(\varepsilon)}$ are isosceles right triangles similar to $\Omega=\left\{\left(x_{1}, x_{2}\right): 0 \leqslant x_{1} \leqslant 1\right.$,

${ }^{4}$ Recall that $\mathcal{N}_{\varepsilon}(x)$ is the $\varepsilon$-neighbourhood of $x$. 
$\left.0 \leqslant x_{2} \leqslant 1 / 2-\left|1 / 2-x_{1}\right|\right\}$. Applying $(6)$, one gets $\mathcal{R}\left(B^{(\varepsilon)}\right)=-\left|\partial\left(\operatorname{conv} B^{(\varepsilon)}\right)\right| \cdot \mathcal{F}(\Omega) \cdot e_{2}=$ $-2 \pi(\sin \varepsilon / \varepsilon) \mathcal{F}(\Omega) \cdot e_{2}$. According to the same formula (6), the resistance of the unit circle $K_{1}$ equals $\mathcal{R}\left(K_{1}\right)=-\left|\partial K_{1}\right| \cdot e_{2}=-2 \pi \cdot e_{2}$. It remains to calculate $\mathcal{F}(\Omega)$.

Let $A_{1}, A_{2}$ and $A_{12}$ be the subsets of $[-\pi / 2, \pi / 2] \times[0,1]$ given by the inequalities $\xi<-\tan \varphi, \xi>1-\tan \varphi$ and $-\tan \varphi<\xi<1-\tan \varphi$, respectively. One easily verifies the following.

(a) If $(\varphi, \xi) \in A_{1}$ then the corresponding billiard particle makes only one reflection from the left leg of $\Omega$ and $\varphi_{\Omega}(\varphi, \xi)=-\pi / 2-\varphi$.

(b) If $(\varphi, \xi) \in A_{2}$ then there is a single reflection from the right leg of $\Omega$ and $\varphi_{\Omega}(\varphi, \xi)=$ $\pi / 2-\varphi$.

(c) If $(\varphi, \xi) \in A_{12}$ then there is a double reflection, either from the left and then from the right leg or vice versa, and $\varphi_{\Omega}(\varphi, \xi)=\varphi$.

Therefore, according to (5), one has

$$
\begin{aligned}
\mathcal{F}(\Omega)=\frac{3}{8} \iint_{A_{1}}(1+\cos (\pi / 2+2 \varphi)) \cos \varphi \mathrm{d} \varphi \mathrm{d} \xi \\
+\frac{3}{8} \iint_{A_{2}}(1+\cos (2 \varphi-\pi / 2)) \cos \varphi \mathrm{d} \varphi \mathrm{d} \xi+\frac{3}{8} \iint_{A_{12}} 2 \cos \varphi \mathrm{d} \varphi \mathrm{d} \xi \\
=I+I I+I I I .
\end{aligned}
$$

A direct calculation gives $I=I I=\frac{3}{4}-\frac{1}{2 \sqrt{2}}, I I I=\frac{3}{2}(\sqrt{2}-1)$; thus, $\mathcal{F}(\Omega)=\sqrt{2}$.

\section{Appendix 2}

Recall that a right triangle is called canonical if (a) it is situated above its hypotenuse and (b) the median dropped on the hypotenuse is vertical. The angle $\alpha$ that the hypotenuse forms with the horizontal line, $\alpha \in(-\pi / 2, \pi / 2)$, is called the inclination of the triangle. Consider a particle that intersects the hypotenuse, gets into the triangle, makes one or two reflections from the legs and then intersects the hypotenuse again and leaves the triangle. Denote by $\varphi$ the angle that the initial velocity $v$ forms with the vector $e_{2}=(0,1)$ and by $\varphi^{+}$the angle that the final velocity $v^{+}$forms with $-e_{2}$. Thus, one has $v=(\sin \varphi, \cos \varphi)$ and $v^{+}=-\left(\sin \varphi^{+}, \cos \varphi^{+}\right)$, where $\varphi$ and $\varphi^{+}$vary between $-\pi / 2+\alpha$ and $\pi / 2+\alpha$.

Parametrize the hypotenuse by the parameter $\xi \in[0,1]$; the value $\xi=0$ corresponds to the left endpoint of the hypotenuse and the value $\xi=1$ to the right one. As in appendix 1, denote by $A_{1}$ the set of values $(\varphi, \xi) \in[-\pi / 2+\alpha, \pi / 2+\alpha] \times[0,1]$ corresponding to particles having single reflections from the left leg, by $A_{2}$ the set of values corresponding to a single reflection from the right leg and by $A_{12}$ the set corresponding to particles having double reflections. One easily finds that $A_{1}$ is given by the inequality $\xi<-\frac{\sin \varphi}{\cos (\varphi-\alpha)}, A_{2}$ by the inequality $\xi>1-\frac{\sin \varphi}{\cos (\varphi-\alpha)}$ and $A_{12}$ by the double inequality $-\frac{\sin \varphi}{\cos (\varphi-\alpha)}<\xi<1-\frac{\sin \varphi}{\cos (\varphi-\alpha)}$. Moreover, for $(\varphi, \xi) \in A_{1} \varphi^{+}=-\pi / 2+\alpha-\varphi$ holds, for $(\varphi, \xi) \in A_{2} \varphi^{+}=\pi / 2+\alpha-\varphi$ holds and for $(\varphi, \xi) \in A_{12} \varphi^{+}=\varphi$ holds. In figure $11, \varphi<0, \alpha>0$ and $\xi_{0}=-\frac{\sin \varphi}{\cos (\varphi-\alpha)}$.

Consider the parallel beam of particles falling on the hypotenuse in the direction $\varphi$. If $|\sin \varphi|<\cos (\varphi-\alpha)$, then the portion of particles that make only one reflection equals $|\sin \varphi| / \cos (\varphi-\alpha)$ and the direction of reflected particles is $\pm \pi / 2+\alpha-\varphi$; one has to choose the sign ' + ' if $\sin \varphi>0$ and ' - ' if $\sin \varphi<0$. The rest of the particles make double reflections; the portion of these particles is $1-|\sin \varphi| / \cos (\varphi-\alpha)$ and the direction of reflected particles is $\varphi$. If $|\sin \varphi|>\cos (\varphi-\alpha)$ then all the particles make a single reflection and the direction of reflected particles is $\pm \pi / 2+\alpha-\varphi$. 


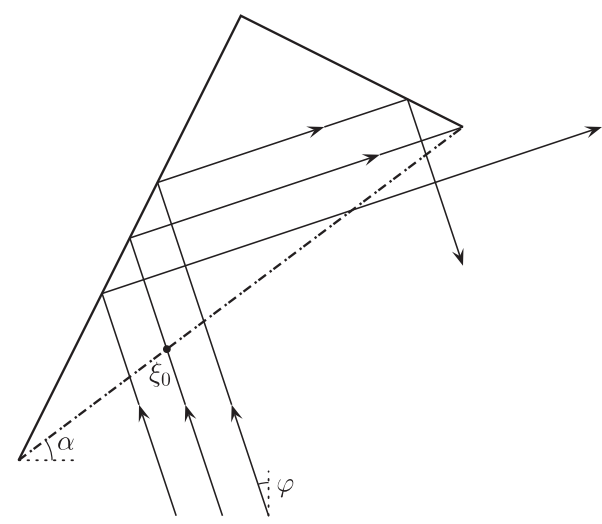

Figure 11. A canonical triangle.

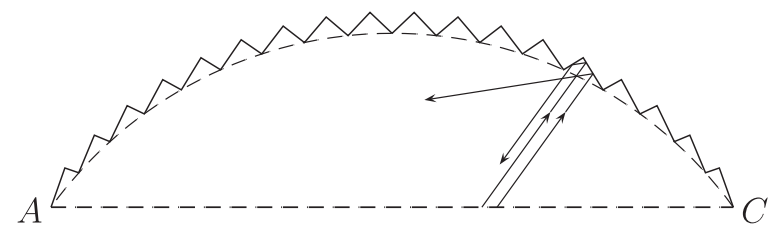

Figure 12. A standard cavity bounded by a canonical broken line.

Now, consider the arc of circumference of angular size $2 \Psi$ contained in the half-plane $x_{2} \geqslant 0$, with the endpoints $A=(0,0)$ and $C=(1,0)$. Parametrize this arc with the parameter $\alpha \in[-\Psi, \Psi]$; the value $\alpha=-\Psi$ corresponds to the point $A$ and $\alpha=\Psi$ to the point $C$. Divide it into a large number of small arcs and substitute each of them with two legs of the corresponding canonical triangle. The resulting broken line (shown in figure 12) defines a standard cavity. Denote by $\delta$ the maximum length of a small arc.

For small $\delta$, the scheme of billiard reflection can be approximately substituted with the following description (pseudo-billiard reflections from the arc $A B C$ ). A particle of some mass moving in a direction $\varphi \in(-\pi / 2, \pi / 2)$ is reflected from the $\operatorname{arc} A B C$. If $|\sin \varphi|<\cos (\varphi-\alpha)$, it is split into two 'splinters' of relative masses $|\sin \varphi| / \cos (\varphi-\alpha)$ and $1-|\sin \varphi| / \cos (\varphi-\alpha)$. The first splinter is reflected in the direction $\pm \pi / 2+\alpha-\varphi$ and the second in the direction $\varphi$. If $|\sin \varphi| \geqslant \cos (\varphi-\alpha)$, there is no splitting, and the whole particle is reflected in the direction $\pm \pi / 2+\alpha-\varphi$. The described dynamics will be called the pseudo-billiard one. A particle of unit mass starts moving at a point of $\mathcal{I}=A C$ in a direction $\varphi \in(-\pi / 2, \pi / 2)$, and after several pseudo-billiard reflections, the resulting splinters return to $\mathcal{I}$.

As a result of the described substitution of the billiard dynamics with the pseudo-billiard one, one obtains the function

$\mathcal{F}(\Psi)=\frac{3}{8} \sum_{i} \int_{-\pi / 2}^{\pi / 2} \int_{0}^{1} m_{i}(\varphi, \xi)\left(1+\cos \left(\varphi_{i}^{+}(\varphi, \xi)-\varphi\right)\right) \mathrm{d} \mu(\varphi, \xi)$,

where $m_{i}=m_{i}(\varphi, \xi)$ are masses and $\varphi_{i}^{+}=\varphi_{i}^{+}(\varphi, \xi)$ the final directions of the splinters resulting from the particle with the initial data $(\varphi, \xi)$. (As we will see later, splitting can really occur only once, after the first reflection; therefore there are at most two splinters.) The difference between $\mathcal{F}(\Psi)$ and the true value of the functional $\mathcal{F}(5)$ is $O(\delta), \delta \rightarrow 0^{+}$. This fact can be expressed as $\lim _{\delta \rightarrow 0^{+}} \mathcal{F}$ (brokenline) $=\mathcal{F}(\Psi)$. Below we will calculate $\mathcal{F}(\Psi)$. 


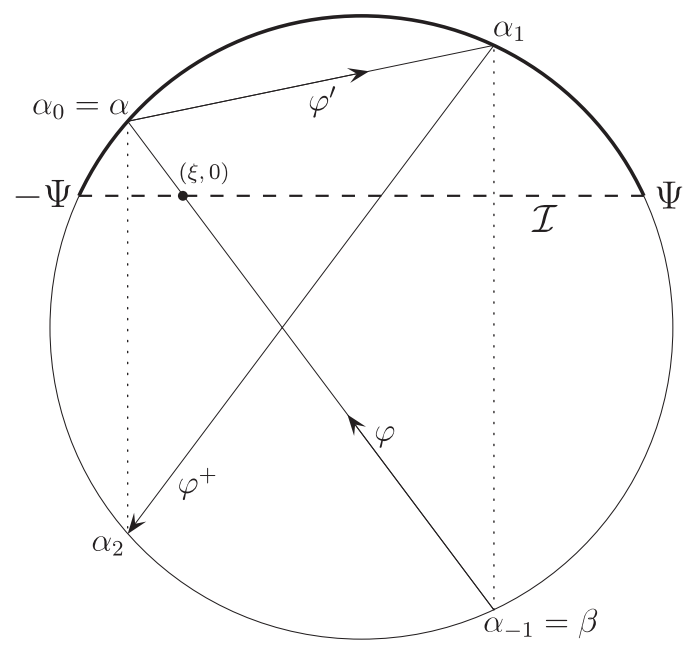

Figure 13. A figure illustrating the pseudo-billiard dynamics.

In order to describe the pseudo-billiard motion, it is helpful to change the variables. Consider the circumference containing the arc under consideration and parametrize it with the same angular variable $\alpha$; this time $\alpha$ varies in $[-\pi, \pi]$. Consider a particle that starts moving at some point $\beta$ of the circumference, intersects $\mathcal{I}$ at some point $(\xi, 0)$ and then reflects from the arc, according to the pseudo-billiard rule, at a point $\alpha$. Thus, one has $\Psi<|\beta| \leqslant \pi$ and $|\alpha|<\Psi$. If $|\beta| \leqslant \pi / 2$, there is no splitting, and if $|\beta|>\pi / 2$, there is.

Let us describe the dynamics of the first splinter. For a while, change the notation; let $\beta=: \alpha_{-1}, \alpha=: \alpha_{0}$ and designate by $\alpha_{1}$ the point of intersection of the splinter trajectory with the circumference. Denote by $\varphi$ the initial direction of the particle and by $\varphi^{\prime}$ the direction of the splinter after the first reflection. (We do not call it $\varphi^{+}$, since there may be more reflections.) One has $\varphi= \pm \pi / 2+\left(\alpha_{0}+\alpha_{-1}\right) / 2$ and $\varphi^{\prime}=\pi / 2+\left(\alpha_{0}+\alpha_{1}\right) / 2$. Then, taking into account that $\varphi^{\prime}= \pm \pi / 2+\alpha_{0}-\varphi$, one gets

$$
\varphi=\left(\alpha_{0}-\alpha_{1}\right) / 2 \text { and }\left(\alpha_{-1}+\alpha_{1}\right) / 2=\pi / 2,
$$

the equalities being true $\bmod \pi$. In other words, the points $\alpha_{-1}$ and $\alpha_{1}$ lie on the same vertical line; see figure 13.

If $\alpha_{1}$ belongs to the arc $[-\Psi, \Psi]$ then there occurs one more reflection, this time without splitting, since the splinter arrived from the point $\alpha_{0} \in[-\pi / 2, \pi / 2]$. Extend the trajectory after the second reflection until the intersection with the circumference at a point $\alpha_{2}$. Using an argument analogous to the one stated above, one derives the formula $\alpha_{0}+\alpha_{2}=\pi$; it follows that the point $\alpha_{2}$ does not belong to the arc, that is, there are no reflections anymore.

Summarizing, the pseudo-billiard dynamics is as follows. After the first reflection from the arc, the particle may, and may not, split into two 'splinters'. If $\alpha_{-1} \in[-\pi / 2,-\Psi] \cup[\Psi, \pi / 2]$, there is no splitting, and the reflection is unique. If $\alpha_{-1} \in[-\pi,-\pi / 2) \cup(\pi / 2, \pi]$, there is splitting into two splinters. If $\alpha_{-1} \in[-\pi+\Psi,-\pi / 2) \cup(\pi / 2, \pi-\Psi]$, the first splinter makes no reflections anymore. If $\alpha_{-1} \in[-\pi,-\pi+\Psi] \cup[\pi-\Psi, \pi]$, it makes one more reflection (without splitting) from the arc, and the final direction is $\varphi^{+}=\pi / 2+\left(\alpha_{1}+\alpha_{2}\right) / 2$. Taking into account the above equalities, one gets $\varphi-\varphi^{+}=\alpha_{-1}+\alpha_{0}+\pi$.

Note that the factor $1+\cos \left(\varphi-\varphi^{+}\right)$, meaning the impact force per unit mass, equals 2 for the second splinter. For the first splinter that makes no reflections, as well as for the reflection 
without splitting, this factor equals $1+\cos \left(\varphi-\varphi^{\prime}\right)=1+\left|\sin \alpha_{-1}\right|$. Finally, for the first splinter that makes one more reflection, this factor equals $1+\cos \left(\varphi-\varphi^{+}\right)=1-\cos \left(\alpha_{0}+\alpha_{-1}\right)$.

Let us pass from the variables $\varphi$ and $\xi$ to $\alpha=\alpha_{0}$ and $\beta=\alpha_{-1}$ and calculate the integral $\mathcal{F}(\Psi)$ in terms of the new variables. The points $\alpha$ and $\beta$ on the circumference have the Cartesian coordinates $\frac{1}{2 \sin \Psi}(\sin \Psi+\sin \alpha,-\cos \Psi+\cos \alpha)$ and $\frac{1}{2 \sin \Psi}(\sin \Psi+\sin \beta,-\cos \Psi+\cos \beta)$, respectively. The interval with the endpoints $\alpha$ and $\beta$ intersects the interval $\mathcal{I}$ at the point $(\xi, 0)$, where

$$
\xi=\frac{\sin (\Psi+\alpha)-\sin (\Psi+\beta)+\sin (\beta-\alpha)}{2 \sin \Psi(\cos \alpha-\cos \beta)} .
$$

Further, one has

$$
\varphi=\frac{\alpha+\beta \pm \pi}{2}
$$

one has to take the sign '-' or ' + ', if $\beta>0$ or $\beta<0$, respectively. Therefore, $\cos \varphi=\left|\sin \frac{\alpha+\beta}{2}\right|$.

Point $(\alpha, \beta)$ runs the set $[-\Psi, \Psi] \times([-\pi,-\Psi] \cup[\Psi, \pi])$, and the mapping $(\alpha, \beta) \mapsto$ $(\varphi, \xi)$ given by (9) and (10) is a one-to-one mapping from this set to $[-\pi / 2, \pi / 2] \times[0,1]$, with the Jacobian

$$
\begin{aligned}
\frac{D(\varphi, \xi)}{D(\alpha, \beta)}= & \frac{\cos (\Psi+\alpha)+\cos (\Psi+\beta)-2 \cos (\beta-\alpha)}{4 \sin \Psi(\cos \beta-\cos \alpha)} \\
& +\frac{\sin (\Psi+\beta)-\sin (\Psi+\alpha)+\sin (\alpha-\beta)}{4 \sin \Psi(\cos \beta-\cos \alpha)^{2}}(\sin \alpha+\sin \beta) \\
& =\frac{1}{4 \sin \Psi} \frac{\sin \frac{\alpha-\beta}{2}}{\sin \frac{\alpha+\beta}{2}}
\end{aligned}
$$

this implies that the integration factor equals

$$
\cos \varphi \mathrm{d} \varphi \mathrm{d} \xi=\frac{1}{4 \sin \Psi}\left|\sin \frac{\beta-\alpha}{2}\right| \mathrm{d} \alpha \mathrm{d} \beta
$$

Further, the mass of the first splinter is $\left|\cos \frac{\alpha+\beta}{2}\right| /\left|\sin \frac{\beta-\alpha}{2}\right|$ and that of the second one is $1-\left|\cos \frac{\alpha+\beta}{2}\right| /\left|\sin \frac{\beta-\alpha}{2}\right|$. Note also that integrating over $\beta \in[-\pi,-\Psi] \cup[\Psi, \pi]$ can be substituted by integrating over $\beta \in[\Psi, \pi]$ with subsequent duplication of the result. With this substitution, one always has $\sin \frac{\beta-\alpha}{2}>0$.

The integral $\mathcal{F}(\Psi)$ can be written down as the sum $\mathcal{F}(\Psi)=I+I I+I I I+I V$, where

$$
\begin{aligned}
& I=\frac{3}{16 \sin \Psi} \int_{-\Psi}^{\Psi} \mathrm{d} \alpha \int_{\Psi}^{\pi / 2}(1+\sin \beta) \sin \frac{\beta-\alpha}{2} \mathrm{~d} \beta \\
& I I=\frac{3}{16 \sin \Psi} \int_{-\Psi}^{\Psi} \mathrm{d} \alpha \int_{\pi / 2}^{\pi} 2\left(\sin \frac{\beta-\alpha}{2}-\left|\cos \frac{\alpha+\beta}{2}\right|\right) \mathrm{d} \beta, \\
& I I I=\frac{3}{16 \sin \Psi} \int_{-\Psi}^{\Psi} \mathrm{d} \alpha \int_{\pi / 2}^{\pi-\Psi}(1+\sin \beta) \cos \frac{\alpha+\beta}{2} \mathrm{~d} \beta, \\
& I V=\frac{3}{16 \sin \Psi} \int_{-\Psi}^{\Psi} \mathrm{d} \alpha \int_{\pi-\Psi}^{\pi}(1-\cos (\alpha+\beta))\left|\cos \frac{\alpha+\beta}{2}\right| \mathrm{d} \beta .
\end{aligned}
$$


As a result of a simple calculation, one obtains

$$
\begin{aligned}
& I=I I I=\frac{3}{16 \sin \Psi}\left[4 \sin \Psi-\frac{8 \sqrt{2}}{3} \sin \frac{\Psi}{2}-\frac{16}{3} \sin ^{4} \frac{\Psi}{2}\right], \\
& I I=\frac{3}{16 \sin \Psi}\left[16 \sqrt{2} \sin \frac{\Psi}{2}-8 \Psi\right], \\
& I V=\frac{3}{16 \sin \Psi}\left[-\frac{8}{3} \sin \Psi+\frac{8}{9} \sin ^{3} \Psi+\frac{8}{3} \Psi\right] .
\end{aligned}
$$

Summing these expressions, one finally comes to formula (7):

$$
\mathcal{F}(\Psi)=1+\frac{1}{6} \sin ^{2} \Psi+\frac{2 \sqrt{2} \sin \frac{\Psi}{2}-2 \sin ^{4} \frac{\Psi}{2}-\Psi}{\sin \Psi} .
$$

\section{References}

[1] Newton I 1687 Philosophiae Naturalis Principia Mathematica (London: Streater)

[2] Buttazzo G and Kawohl B 1993 On Newton's problem of minimal resistance Math. Intell. 15 7-12

[3] Brock F, Ferone V and Kawohl B 1996 A symmetry problem in the calculus of variations Calc. Var. 4 593-9

[4] Buttazzo G, Ferone V and Kawohl B 1995 Minimum problems over sets of concave functions and related questions Math. Nachr. 173 71-89

[5] Buttazzo G and Guasoni P 1997 Shape optimization problems over classes of convex domains J. Convex Anal. 4 343-51

[6] Lachand-Robert T and Peletier M A 2001 Newton's problem of the body of minimal resistance in the class of convex developable functions Math. Nachr. 226 153-76

[7] Lachand-Robert T and Peletier M A 2001 An example of non-convex minimization and an application to Newton's problem of the body of least resistance Ann. Inst. Henri Poincaré, Anal. Non Lin. 18 179-98

[8] Comte M and Lachand-Robert T 2001 Newton's problem of the body of minimal resistance under a single-impact assumption Calc. Var. Partial Diff. Eqns. 12 173-211

[9] Comte M and Lachand-Robert T 2001 Existence of minimizers for Newton's problem of the body of minimal resistance under a single-impact assumption J. Anal. Math. 83 313-35

[10] Lachand-Robert T and Oudet E 2006 Minimizing within convex bodies using a convex hull method SIAM J. Optim. 16 368-79

[11] Plakhov A Yu 2003 Newton's problem of a body of minimal aerodynamic resistance Dokl. Akad. Nauk 390 314-17

[12] Plakhov A Yu 2003 Newton's problem of the body of minimal resistance with a bounded number of collisions Russ. Math. Surv. 58 191-2

[13] Plakhov A Yu 2004 Newton's problem of the body of minimum mean resistance Sbornik: Math. 195 1017-37

[14] Bunimovich L B 2001 Mushrooms and other billiards with divided phase space Chaos $11802-8$ 